\title{
PROKLA-Redaktion
}

\section{Editorial: Wissen und Eigentum im digitalen Zeitalter}

In dem Maße, in dem sich der Kapitalismus in der Neuzeit als herrschende Produktionsweise durchsetzte, löste er die vielfältigen, in unterschiedliche soziale Kontexte eingebundenen Besitz- und Nutzungsverhältnisse in eindeutige private oder öffentliche Eigentumsverhältnisse auf. Dieser Prozess erfasste zunächst materielle Dinge, Boden sowie die individuelle Arbeitskraft. Der heute ganz selbstverständliche Eigentumsanspruch auf Gedanken, Ideen, Informationen aller Art, das Recht auf „geistiges Eigentum", setzte sich jedoch erst relativ spät durch. Damit war der Kapitalverwertung einerseits ein neues Feld eröffnet, andererseits aber auch Schranken auferlegt, indem „Wissen“ keine frei verfügbare Ressource mehr darstellte.

Die Institution des geistigen Eigentums, differenziert in verschiedene Rechtsinstrumente wie das Urheberrecht, das Patentwesen, den Markenschutz etc., gibt den Wissensproduzenten oder ihren Verwaltern das Mittel in die Hand, geistige Schöpfungen handel- und vermarktbar zu machen, wobei die exklusiven Eigentumstitel nur den zahlungsfähigen Individuen Zugang zum Wissen eröffnen, während die nicht-zahlungsfähigen in der Regel vom Zugang ausgeschlossen werden. Bereits bei Bildung und Ausbildung erweist sich dieser Ausschluss aber als dysfunktional für die kapitalistische Produktionsweise. Hier obliegt es dem Staat, die radikalen Ausschlussmechanismen des kapitalistischen Privateigentums zu kompensieren. Das staatliche Bildungssystem ist (oder eher: war) Mittel dieses Ansinnens.
Der staatlich alimentierte Zugang der Menschen zu Wissen wird in historisch wechselndem Ausmaß für nötig gehalten - moralische Universalwerte („Bildung ist ein Menschenrecht") spielen dabei eine allenfalls untergeordnete Rolle.

Die Widersprüchlichkeit der Exklusivität des Wissens - einerseits ist das privateigentümliche Wissen Mittel der Kapitalverwertung, andererseits eine seiner Grenzen - ist aber nicht nur für das Ausbildungssystem von entscheidender Bedeutung, sondern auch für die Verwertungsstrategien der einzelnen Kapitale, was in früheren Jahrzehnten bereits im Streit um die Dauer von Patentrechten deutlich wurde. Dieses grundsätzliche Problem hat durch die neuesten technischen Entwicklungen eine dramatische Zuspitzung erfahren. Digitalisierung der Information und Internet ermöglichen heute eine praktisch kostenlose und von jedermann zu bewerkstelligende Vervielfältigung und weltweite Distribution von „Wissen“ - worin Texte, Bilder, Musik und Algorithmen jeder Art eingeschlossen sind. Das Privateigentum an Wissensprodukten scheint sich aus technischen Gründen kaum noch aufrecht erhalten zu lassen. Gleichzeitig nimmt der Anteil an Wissensprodukten gegenüber materiellen Produkten im gegenwärtigen Kapitalismus aber beständig zu. Wissen wird zum einen verstärkt zum hoffnungsbeladenen Vehikel der Mehrwertproduktion und seine Sicherung als Privateigentum aus Sicht der entsprechenden Akteure daher notwendigerweise immer umfassender und restriktiver. 
Zum anderen ist diese Sicherung aber nicht nur zunehmend schwieriger durchsetzbar, sie kann auch als Hemmnis der Kapitalverwertung (etwa aufgrund steigender Transaktionskosten) oder weiterer Produktivkraftentwicklung in Erscheinung treten.

Der Privateigentumscharakter von Wissen gilt in dieser Situation nicht mehr unbedingt als selbstverständlich, er wird zu einem gesellschaftlich umkämpften Verhältnis. Die Vertreter einer Verschärfung des traditionellen „Copyright" treffen auf den erbitterten Widerstand der Verfechter des „Copyleft“. Dass diese beiden sich scheinbar diametral gegenüber stehenden Positionen mehr gemeinsam haben als sie wissen, wird von Sabine Nuss in ihrem Beitrag gezeigt: Letzten Endes gründen beide Auffassungen - so die These - in derselben bürgerlichen Eigentumskonzeption.

Eines der bekanntesten Beispiele für neue Produktions- und Eigentumsformen, die auf den neuen Möglichkeiten des Internet gründen, ist die Freie Software mit dem Betriebssystem Linux als Vorzeigemodell. Bei Freier Software werden die Produkte unter Verzicht auf Privateigentum am zugrundeliegenden Wissen (dem Quellcode des Programms) weltweit, kooperativ, meistenteils unbezahlt und via Internet koordiniert hergestellt. Inzwischen ist Freie Software über die internen Kreise der Computerspezialisten hinaus bekannt geworden und dient zum einen als Projektionsfläche für alternative Gesellschaftsentwürfe. Zum anderen ist inzwischen auch die Industrie aufmerksam geworden und engagiert sich für den Einsatz und die Entwicklung von Freier Software. Warum das so ist, untersucht Werner Winzerling. Unterschiedliche Reaktionsweisen auf die Digitalisierung des Wissens werden von Rainer Kuhlen analysiert. Er stellt der „Napsterisierung“ (in Anlehnung an die Musiktauschbörse Napster) die „Venterisierung“ (in Anlehnung an Craig Venter, Gründer der Firma Celera Genomics, die maßgeblich an der Entschlüsselung des menschlichen Genoms beteiligt war) gegenüber.

Dass der Kapitalismus nicht grundsätzlich in Frage gestellt wird, wenn Teile der Produktionsmittel oder -ergebnisse frei von exklusiver Verfügungsgewalt bleiben, wird nicht nur bei der Integration Freier Software in kapitalistische Verwertungszusammenhänge deutlich. Bezüglich des Patentwesens gibt es auch innerhalb der herrschenden ökonomischen Theorien zunehmend dezidierte Kritik an der Monopolisierung von Wissen, wonach Patente entgegen landläufiger Meinung innovationshemmend seien. Über „kapitalistische Eigentumskritik im Patentwesen" schreibt Boris Gröndahl.

Verwertung ist nie auf den nur nationalen Rahmen beschränkt gewesen, dies gilt auch für "Wissensprodukte“. Voraussetzung dafür ist aber die Durchsetzung und Standardisierung eigentumssichernder Institutionen, was für geistiges Eigentum insbesondere im Rahmen der WTO mit dem Abkommen über TRIPS (Trade Related Aspects of Intellectual Property Rights) angestrebt wird. Dass die Sicherung geistigen Eigentums auf der einen (nördlichen) Seite der Welt zugleich Enteignung auf der anderen (südlichen) Seite bedeutet, dass damit der Nord-Süd Konflikt nochmals verschärft wird, zeigt der Beitrag von Michael Frein.

Mit der historischen Herausbildung von geistigen Eigentumsrechten beschäftigt sich Jeanette Hofmann. Sie zeigt auf, dass es nicht nur spezifische Verwertungsinteressen waren, sondern auch ein bestimmtes bürgerlich-individualistisches Menschenbild, das im Geniekult des 18. Jahrhunderts kulminierte, welche die uns heute so vertraute Institution des geistigen Eigentums hervorbrachten.

Außerhalb des Schwerpunkts beschäftigt sich Susanne Hildebrandt mit der Privatisierung des Gemeindelandes in Mexiko, die verstärkt in den 90er Jahren einsetzte und dort noch für erhebliche soziale Konflikte sorgen dürfte. 